\title{
XXXVI. On diffraction-colours, with special reference to coronœ and iridescent clouds
}

\author{
James C. McConnel M.A.
}

To cite this article: James C. McConnel M.A. (1889) XXXVI. On diffraction-colours, with special reference to coronœ and iridescent clouds, Philosophical Magazine Series 5, 28:173, 272-289, DOI: $10.1080 / 14786448908621356$

To link to this article: http://dx.doi.org/10.1080/14786448908621356

曲 Published online: 08 May 2009.

Submit your article to this journal $[\pi$

Џll Article views: 2

Q View related articles $\square$ 
For the same substance the right-hand side should be a constant, and it has been shown that it nearly fulfils this condition.

Mr. Dale now states that for different substances

$$
\log \frac{\mu-1}{\delta}=a c \text {, }
$$

where $c$ is a constant independent of the nature of the substance, and $a$ is an integer. This at once leads to the relation $v / m=\frac{2}{3} e_{n e}$, which would indicate that the ratios of the volumes to the masses of the molecules are in geometrical progression, or, more shortly, the densities of the molecules are in geometrical progression.

If, then, Mr. Dale's conclusions are correct this would be the theoretical inference to be drawn from them.

XXXVI. On Diffraction-Colours, with special reference to Corone and Iridescent Clouds. By JAMes C. M $\mathrm{M}^{\circ} \mathrm{ConnEL}$, M.A., Fellow of Clare College, Cambridge*.

[Plate X.]

T $N$ a previous paper $\dagger \mathrm{I}$ have explained the occurrence of 1 bright colours in certain clonds near the sun on the hypothesis that the light is diffracted by thin needles of ice or by fine drops of water. In the present paper I give a more complete determination of the actual colours produced, based on Maxwell's observations of the colour-relations of the solar spectrum. The first section contains a mathematical investigation of the light diffracted by clouds of filaments and of spherules respectively. The second is devoted to calculating the colours and setting them out on Maxwell's diagram. And I have been tempted to mark also on the diagram the colours of the sky and sun. In a fature number of this Magazine I hope to publish some additional remarks on iridescent elouds and allied phenomena, including Bishop's ring.

\section{Mathematical Expressions. \\ Rectangular Aperture. Point Source.}

We will first take the case of a rectangular aperture (sides $a b$ ) in an opaque diaphragm inclined to the incident light at an angle $\gamma$, and discuss the illumination on a spherical screen of very large radius $f$, whose centre 0 coin-

* Communicated by the Author.

$\dagger$ Phil. Mag. November $188 \pi$. 
with special reference to Corone and Iridescent Clouds. 273 cides with that of the aperture. The side $a$ is at right angles to the incident light. In fig. 1 only a quarter of the aperture is represented.

$\mathrm{O}$ is the origin of coordiuates; the axis of $z$ is drawn towards the source of light, while the axis of $x$ is parallel to the side $a$ of the aperture.

$\mathrm{P}(x y z)$ is a point in the aperture.

$\mathrm{M}(\xi \eta \zeta)$ a point on the spherical screen.

The source of light is small and very distant.

Let the vibration at $O$ be represented by $\cos \kappa v t$, where $t$ is the time, $v$ the velocity, and $\kappa=2 \pi / \lambda$. The intensity is then unity. The vibration at $\mathrm{P}$ is $\cos \kappa(v t+z)$.

Fig. 1.

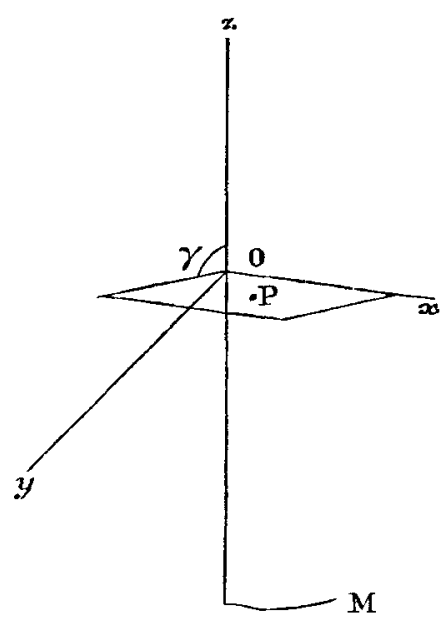

We now break up the primary wave into its secondary components over the plane of the aperture, which is not a wave-front. The disturbance at $\mathrm{M}$ due to the element $d x d y$ at $\mathrm{P}$ is

where $\rho=$ MP.

$$
-\frac{d x d y}{\lambda \rho} \sin \kappa(v t+z-\rho)^{*}, \quad . \quad . \quad . \quad \text {. }
$$

Now

$$
\rho^{2}=(x-\xi)^{2}+(y-\eta)^{2}+(z-\zeta)^{2}, \text { and } \xi^{2}+\eta^{2}+\zeta^{2}=f^{2} ;
$$

$x y z$ are small compared with $\xi \eta \zeta$; so, neglecting their squares, we bave

$$
\rho^{2}=f^{2}-2 x \xi-2 y \eta-2 z \zeta \text {. }
$$

In the last term we can put $\zeta=-f$, and obtain

$$
\rho=z+f\left(1-\frac{x \xi+y \eta}{f^{2}}\right) \text {. }
$$

In the denominator of (1) we may write $\rho=f$. So the vibration at $M$ is

$$
-\frac{1}{\lambda f} \iint \sin \kappa\left(v t-f+\frac{x \xi+y \eta}{f}\right) d x d y
$$

the limits of $x$ and $y$ being $\pm \frac{a}{2}$ and $\pm \frac{b \sin \gamma}{2}$ respectively.

* Encyc. Brit., art. "Wave Theory of Light," p. 429 . 
Hence, as usual, the illumination on the screen is given by

$$
I=\frac{a^{2} b^{2} \sin ^{2} \gamma}{f^{2} \lambda^{2}} \frac{\sin ^{2} \frac{\pi a \xi}{f \lambda} \sin ^{2} \frac{\pi b \sin \gamma \eta}{f \lambda}}{\frac{\pi^{2} a^{2} \xi^{2}}{f^{2} \lambda^{2}}} \frac{\pi^{2} b^{2} \sin ^{2} \gamma \eta^{2}}{f^{2} \lambda^{2}} .
$$

The field is crossed by two sets of parallel dark bands, given by $a \xi / f \lambda$ and $b \sin \gamma \eta / f \lambda$ being positive or negative integers other than zero. There is a large central rectangle of dimensions $2 f \lambda / a$ by $2 f \lambda / b \sin \gamma$, surrounded by others of similar shape but only a quarter the size. The brightest point is the centre, and along the two principal directions the successive maxima have approximate values $0.046,0.017,0.0085$, $0.0050 \ldots$, that at the centre being unity, and are found at distances given by

$$
a \xi / f \lambda=1 \cdot 43,2 \cdot 46,3 \cdot 47,4 \cdot 47 .
$$

Along the diagonals the numbers for the successive maxima are the squares of the above, viz. $0.0021,0.00029,0.00007 \ldots$, so the diminution is much more rapid.

It is obvious from (2) that the linear dimensions of the diffraction-pattern are proportional to $\lambda$, and its brightness proportional to $\lambda^{-2}$. Thus, if the sonrce send out white light, the central patch will be bluish in the middle and tinged with yellow and red at its edges. Along one of the principal directions, e.g. $\eta=0$, we have, governing the colour of the light, the factor

$$
\sin ^{2} \frac{\pi a \xi}{f^{\prime} \lambda}
$$

As will be explained in the next section, the colours are those of Newton's rings, though their relative brightness is very different. Along the diagonals for which $a \xi=b \sin \gamma \eta$, the colour-factor is

$$
\lambda^{2} \sin ^{4} \frac{\pi a \xi}{f \lambda} \text {. . . . . . . . }
$$

So that the tints are much richer, though the intensity is very feeble.

$$
\text { Babinet's Principle. }
$$

Suppose now that $b \sin \gamma$ is much greater than $a$, so that our aperture becomes a narrow slit. We are intending to deal, not with slits in an opaque screen, but with filaments in the open sky. This case may be immediately derived from the other with the aid of Babinet's principle. But it is desirable, I think, to examine the application of the principle in some detail. Replace the slit by a very much larger aperture, say 500 times as long and 10,000 times as broad. The 
with speciul reference to Coronce and Iridescent Clouds. 275

screen is so far away that our expression (2) still applies ; in other words, the diffraction-pattern is not supplanted by a geometrical image of the aperture. But the diffractionpattern is enormously reduced in size, and outside it there is no light. In this outer region the illumination will be the same whether we block up all the aperture except the original slit, or block up the slit by an opaque filament leaving the rest of the aperture open. For the two portions of light must be able to neutralize each other. So we may replace the slit by a filament of thickness $a$ and length $b$, inclined to the incident light at the angle $\gamma$, lying within our large aperture, and (2) will still hold good except within a negligibly small area. If then, according to (3), at any point in the screen the light, is, say, green, to an eye placed at that point the aperture will appear. a green speck. As the green light is not in any way altered by increasing the size of the aperture, it is clear that it must come from the region immediately surrounding the filament, and that the filament will look green even when the diaphragm is entirely removed.

\section{Cloud of Filaments.}

The effect then is the same, whether it be produced by slits in an opaque diaphragm or by filaments in an open space. The calculations are simpler in the case of slits, but practically we have to deal with filaments. So in future we shall speak of filaments only, and in treating of the illumination of the screen we shall refer only to the diffracted light and ignore that which comes direct.

As $a$ is made small compared with $b \sin \gamma$, the diffractionpattern is stretched ont into a long strip, very narrow in the $y$ direction. If there be a large number $n$ of filaments equally inclined to the axis of $z$ and evenly distributed round it, the illumination is found by summing the illuminations due to the individuals. Practically we have to distribute the light we find, according to (2), on any circle round the axis of $z$ evenly over the whole circle and then multiply it by $n$. Owing to the narrowness of the strip we can treat $\xi$ as constant for points on the cirele where the illumination is sensible. So of the three factors on the right-hand side of (2), it is only the last that varies. This third factor may be written $p=\sin ^{2} u / u^{2}$, where $u=\pi b \sin \gamma \cdot \eta / f \lambda$. If $\xi$ and $-\xi$ be the points where the circle cuts the plane $y=0$, the average value of $p$ over the whole circle is, remembering that the strip is cut twice,

$$
\int p d \eta / \pi \xi \text {. }
$$

The integration extends over the region for which $p$ is sensible, and we are of course at liberty to extend the limits to $\pm \infty$. 
So

$$
\begin{gathered}
\text { Now } \int_{-\infty}^{+\infty} \frac{\sin ^{2} u}{u^{2}} d u=\pi \text { is a known result, and } \\
\pi b \sin \gamma d \eta / f \lambda=d u \text {. }
\end{gathered}
$$

Hence the illumination on the screen at points in the plane $y=0$, due to a large number $n$ of equal regularly distributed filaments making an angle $\gamma$ with the axis of $z$, is by (2),

$$
\frac{n a^{2} b \sin \gamma}{\pi f^{2} \lambda} \frac{f}{\xi} \frac{\sin ^{2} \frac{\pi a \xi}{f \lambda}}{\frac{\pi^{2} a^{2} \xi^{2}}{f^{2} \lambda^{2}}} . . . . . .
$$

To extend this to the case when the filaments are uniformly distributed in all directions we must replace $n b \sin \gamma$ by $\Sigma b \sin \gamma$. If they occupy the fraction $\alpha$ of the field of view looking from the screen, and the summation be extended over an angular area $\omega$ equal to that of the sun, we have

$$
a \omega f^{2}=\Sigma a b \sin \gamma=a \Sigma b \sin \gamma .
$$

Now the direct illumination of the sun at the cloud is, by hypothesis, unity, and it has the same value where the observer stands, $i$. e. at the imaginary screen. And it is obvious that the apparent brightness of the sun and cloud are in the same ratio as the illumination due to equal angular areas of each. So, finally, the brightness of the cloud of filaments is in terms of that of the sun,

$$
\alpha \omega \frac{a f}{\pi \xi \lambda} \frac{\sin ^{2} \frac{\pi a \xi}{f \lambda}}{\frac{\pi^{2} a^{2} \xi^{2}}{f^{2} \lambda^{2}}} . \text {. . . . . . . }
$$

On p. 431 of my former article are given expressions for the brightness of the first, second, and fourth bright rings in a cloud of filaments, obtained in a different manner. It will be found on examination that these expressions agree with (6).

In a cloud of filaments, of diameter $a$, the first four maxima, according to (6), are proportional to $1,0.215,0.076,0.035$; the ninth being 0.0036 , and the central maximum being infinite. This last result is not surprising, for we have supposed, throughout the greater part of the argument, the source of light to be indefinitely small.

In sunlight the colour is defined by the factor

$$
\lambda \sin ^{2} \frac{\pi a \xi}{f \lambda} \text {. . . . . . . . . }
$$

These are the colours produced when the source is a luminous 
with special reference to Coronce and Iridescent Clouds. 277

line and the diffracting aperture a parallel slit, for the blurring is of the same nature as that involved in the transition from (2) to (5).

\section{Diagonals of Square Aperture.}

Before giving the accurate expression for the illumination when the diffracting aperture is circular, it will be instructive to examine a case which presents the same peculiarities in an exaggerated form. The main difference between a circle and a square, as regards diffraction in directions parallel to the sides of the square, is that in the former the outlying portions, where the phase-difference is greatest, are relatively small. This feature is still more marked in diffraction by a square parallel to its diagonals (see fig. 2), and this is a case we have incidentally solved.

Let $c$ be the diagonal of the square, and $\zeta$ the distance of the point on the screen from the centre of the figure in a direction parallel to the diagonal. Then, putting $\gamma=\pi / 2$ in (2), we obtain

$$
I=\frac{c^{4}}{4 f^{2} \lambda^{2}} \frac{\sin ^{4} \frac{\pi c \zeta}{2 f \lambda}}{\frac{\pi^{4} c^{4} \zeta^{4}}{16 f^{4} \lambda^{4}}} . . . . . .
$$

The dark points are given by $c \xi=2 m f^{\prime} \lambda$, where $m$ is any integer other than zero; and in general corresponding points are twice as far out as in directions parallel to the sides of a square of side $c$. As we bave already seen, the diminution of brightness is much more rapid, and the colours, when sunlight is used, are purer.

\section{Circular Aperture.} is

The expression corresponding to (2) for a circular aperture

where

$$
\mathrm{I}=\frac{\pi^{2} \mathrm{R}^{2}}{\lambda^{2} f^{2}} \frac{4 \mathrm{~J}_{1}^{2}(z)^{*}}{z^{2}} ; \text {. . . . . . . . }
$$

$\mathrm{R}$ is the radius of the aperture, and $r$ the distance on the screen from the centre of the diffraction-figure. The dark

$$
\text { * 'Wave Theory,' p. } 432 .
$$


rings are given by

$$
z / \pi=1 \cdot 22, \quad 2 \cdot 23, \quad 3 \cdot 24, \quad 4 \cdot 24, \ldots
$$

and the maxima of the bright rings are given by

$$
z / \pi=1 \cdot 63,2 \cdot 68, \quad 3 \cdot 71,4 \cdot 72, \ldots
$$

and have the values

$$
0.0175,0.00416,0.00160,0.00078 \text {, }
$$

that at the centre being unity. Thus corresponding parts are rather further out than in the principal directions for a square of side $2 \mathrm{R}$, and the brightness falls off much more rapidly. It seems legitimate to assume that the colours also, when sunlight is used, are slightly purer.

\section{Cloud of Water-drops.}

To pass from the illumination on a screen, due to a single circular aperture, to the brightness of a water-cloud, we follow the lines of the previous argument, with, however, considerable simplification, owing to the orientation of a sphere being a matter of indifference. We have to multiply (y) by the number $n$ of drops within an angular area equal to that of the sun, and this number is given by

$$
n \pi \mathrm{R}^{2}=\alpha f^{2} \omega \text {. }
$$

So the brightness of the cloud, in terms of that of the sun, is

$$
\alpha \omega \frac{\pi \mathrm{R}^{2}}{\lambda^{2}} \frac{4 \mathrm{~J}_{1}^{2}(z)}{z^{2}} \text {. . . . . . . }
$$

The remarks we bave made on (9) apply equally well to (10). The colour-factor in both cases is $\mathrm{J}_{1}^{2}(z)$. For the two kinds of clouds, compare the values of the maxima given under (6) and under (9). We are enabled to make a fairly complete comparison by the following result. When $z$ is great,

giving a colour-factor

$$
J_{1}^{2}(z)=\frac{2}{\pi z} \sin ^{2}\left(z-\frac{\pi}{4}\right) \text { nearly*, }
$$

$$
\lambda \sin ^{2}\left(z-\frac{\pi}{4}\right) .
$$

Even at the first bright ring the approximation is fair, for it gives the first maximum at $z=1.716 \pi$ with the value 0.0162 ; and it rapidly improves as $z$ increases, though always better at the maxima than at intermediate points. The expression (6) may be written in the form

$$
\begin{gathered}
\alpha \omega \frac{a^{2}}{\lambda^{2} z_{1}} \frac{\sin ^{2} z_{1}}{z_{1}^{2}}, \\
\text { * 'Wave Theory,' p. } 432 .
\end{gathered}
$$


with special reference to Corona and Iridescent Clouds. 279

where

$$
z_{1}=\pi a \xi / f \lambda \text {. }
$$

To secure corresponding points, let

$$
z_{1}=z-\frac{\pi}{4}
$$

and let us choose such values of $\mathrm{R}$ and $a$ that the angular distance from the sun is the same $(=\theta)$ in both cases. For this,

$$
a \theta=2 \mathrm{R} \theta-\lambda / 4 ;
$$

and, since by hypothesis $z$ is great, $a \theta$ amounts to several wave-lengths; and this equation is satisfied by nearly the same values of $a$ and $2 \mathrm{R}$ throughout the visible spectrum. The ratio of the brightness of a water-cloud to that of an icecloud is then $8 \mathrm{R}^{2} z_{1}^{3} / a^{2} z^{3}$, or $2 z_{1} / z$. Thus the outlying spectra from water-drops are about twice as bright as those from icefilaments, when the drops and the filaments occupy the same fraction of the field of view, and corresponding spectra are at the same distance from the sun.

\section{Influence of Transparency.}

We have incidentally assumed that drops of water and needles of ice can be treated as opaque objects. Now if a single hexagonal filament be placed with one of its faces normal to the sun's rays, it is clear that the light, transmitted through the part that behaves like a parallel plate, must interfere with the light that passes on either side of the filament, and should be taken into account. But with the varying orientation of the filaments, the quantity and relative retardation of the transmitted light would alter to such an extent that the practical result in the case of a cloud would merely be the addition of so much white light.

The case of a spherical drop of water does not admit of the same variety. And it would seem that, when the size is uniform, the transmitted light should be taken into account. The investigation would be complex, even if it be possible with our present knowledge; but we see at once that a large part of the light must be retarded relatively to the uninterrupted light by about a third of the diameter of the drop. Thus the character of the effect would change completely with small changes in the size, and in ordinary clouds we shall not be far wrong in treating the drops as opacue. It is probable that the comparative poorness of water iridescences is partly due to this cause.

The legitimacy of adding the illuminations due to the different drops, without reference to phase, has been shown by 
Lord Rayleigh to depend on "the light being heterogeneous, the source of finite area, and the obstacles in motion."

\section{Curves on the Colour Diagram.}

In a very interesting paper (Trans. Roy. Soc. Edinb. July 1886) Lord Rayleigh has set out a curve representing the series of colours of thin plates on Maxwell's form of Newton's diagram. Before such a calculation had been made, it would have been impossible to predict from theory, except in the very roughest manner, the nature of these colours, though the exact composition of the light in terms of wave-lengths were thoroughly known. The reading of this paper made me anxious to obtain a more complete theory of the splendid colours of iridescent clouds, and I have incidentally determined some of the colours of various diffraction-patterns. This led to the discovery of a serious blunder which I made in my former paper on iridescent clouds, in supposing that the central band in the diffraction-pattern of a slit was colourless. I was following high authority, for Verdet says (Leçons d" Optique Physique, $\$ 70$ ), "on aperçoit au centre du phénomène une bande blanche et brillante, qui est située sur la direction normale à la fente diffringente." As soon as attention is called to the matter, it is obvious that the edge must be reddish, since the breadth of the band in homogeneous light is proportional to the wave-length; and, as a matter of fact, this red fringe is the finest red of the whole series. The centre of the band is a pale though bright blue. But this depends on the introduction of the factor $\lambda^{-1}$ in the expression for the secondary vibration, the necessity for which was not recognized in Verdet's time. I do not remember seeing the coloration of the central band distinctly pointed out, though it is implicitly contained in a statement of Verdet in the very section I have quoted. He says that, when white light is used, the red bands correspond to the absence of the brightest part (i. e. the yellow) of the spectrum. This is not quite correct, for my results show that they correspond to the absence of the blue-green.

As the three corners of his diagram, Maxwell ("Theory of Compound Colours," Phil. Trans. March 1860) selected equal widths on his prismatic spectrum, near the points marked on his scale by 24,44 , and 68 . Between any colour whatever and these three a match can be made by altering the proportions ; either a combination of three matching the remaining one, or a combination of two matching a combination of the other two. Thus, for example, unit width at any point of his prismatic spectrum could be expressed as the sum of multiples 
with special reference to Coronce and Iridescent Clouds. 281

of the three units, using negative signs when required. Looking at the table below, we see

$$
(36)=0 \cdot 48(24)+1 \cdot 25(44)-0.02(68) \text {; }
$$

which means that the mixture of 0.02 of (68) with unit width of (36) is indistinguishable in hue, depth, or brightness from a mixture of 0.48 of (24) with 1.25 of (44). The position of (36) on the diagram is the centre of gravity of weights proportional to $0.48,1.25$, and -0.02 , placed at the three corners (24), (44), and (68). The brightness of a colour is not indicated by the diagram.

The most important property of the diagram is the following. Let us define the brightness of any colour to be the algebraic sum of the corresponding multiples of the three corner units. Then if any colour $\mathrm{C}$ be composed of the colour $A$ of brightness $\alpha$, and of the colour B of brightness $\beta$, its position on the diagram is the centre of gravity of weights $\alpha$ at $A$, and $\beta$ at $B$, and its brightness is $\alpha+\beta$. Hence all the colours on any straight line are mixtures of the colours at the two ends of the line, and, in particular, all the points on a straight line drawn from the point white are of the same hue; the depth or purity increasing as we near the spectrumcolours on the borders of the diagram.

Now Maxwell has determined the multiples necessary to express unit width at any point of the spectrum in terms of the three corner units. The sum for the whole spectrum must represent white. And, if the relative brightness of different parts of the spectrum be altered in a known manner, we can, by introducing the proper factors before summation, find the resultant colour in terms of the three units. Isord Rayleigh used a table, containing twenty-two equidistant points of the spectrum, based on Mrs. Maxwell's observations. From this I have deduced the following abridged table, which is sufficiently accurate for my purpose :-

\begin{tabular}{|c|c|c|c|c|}
\hline Scale-number. & Wave-lengtb. & $(24)$ & $(44)$ & (68) \\
\hline \multirow[t]{2}{*}{$\begin{array}{l}20 \\
28 \\
36 \\
44 \\
52 \\
60 \\
68 \\
76 \\
84 \\
92\end{array}$} & \multirow[t]{2}{*}{$\begin{array}{l}663 \\
608 \\
563 \\
529 \\
500 \\
475 \\
457 \\
441 \\
428 \\
417\end{array}$} & $\begin{array}{c}+0.44 \\
+1.17 \\
+0.48 \\
\ldots \ldots \\
-006 \\
-0.05 \\
\ldots \ldots . \\
+0.03 \\
\ldots \ldots \\
\ldots \ldots\end{array}$ & $\begin{array}{c}+0.32 \\
+1.25 \\
+1.00 \\
+0.51 \\
+0.19 \\
\ldots \ldots . . \\
\ldots \ldots . \\
\ldots \ldots . . \\
\ldots . .\end{array}$ & $\begin{array}{l}+0.04 \\
-0.02 \\
+0.28 \\
+0.75 \\
+1.00 \\
+0.69 \\
+0.33 \\
+0.15\end{array}$ \\
\hline & & +201 & $+3 \cdot 27$ & $+3 \cdot 2 \cdot 2$ \\
\hline
\end{tabular}


The white obtained by superimposing unit widths at the ten points is given by

$$
\mathrm{W}=2 \cdot 01(24)+3 \cdot 27(44)+3 \cdot 22(68) \text {. }
$$

The chief defect of this table is the omission of the red corner (24). This has been in great measure allowed for by modifying the coefficients for (20) and (28). At the same time the white was brought to practical coincidence with the white of Lord Rayleigh's table.

On the diagram (PI. X.) are marked the positions of sixteen points equidistant in the prismatic spectrum, from 20 to 80 on Maxwell's scale, with the corresponding wave-lengths. These lie, for the most part, outside the triangle. Rood has determined the places in the spectrum which, when diluted with a suitable amount of white, match the colours of certain pigments ('Modern Chromaties,' p. 38). I had no data for marking the true position of the pigments on the diagram, but their bues (i.e. the radii from white on which they lie) are indicated. I have also divided the diagram into five parts, blue, green, yellow, red, and purple, chiefly in order to name the hues in the "brilliancy" curves described below. In this I have been mainly guided by Rood's 'Modern Chromatics.' On the spectral colours his statements are definite. But the limits of purple, founded on considerations of complimentary colours, are more doubtful. The estimation of hue depends greatly on the brightness of the light and the purity of the colour; and of course, at the best, the lines of division must be rather indefinite. The pure yellow in the spectrum is a very narrow band; so my yellow division consists mainly of orange-yellow and greenish yellow.

In the previous section I have shown that to find the colours in the principal directions of the diffraction-pattern of a rectangular aperture, the proper factor to multiply each of the constituents of sunlight before compounding them is $\sin ^{2} \frac{\pi a \xi}{f \lambda}$. For the colours of thin plates the appropriate factor, "strictly applicable only to a plate of air bounded by media of small refrangibility," but practically sufficient for all ordinary cases, is $\sin ^{2} \frac{\pi \mathrm{V}}{\lambda}$. Thus identical colours are found in the two cases, whenever the "retardation " $V$ for the thin plate is equal to the extreme retardation $a \xi / f$ of light from one edge of the aperture relative to light from the other. The dotted curve (copied from Lord Rayleigh's) represents these colours, and the small figures at the side are values of $a \xi / f$ expressed, like the wave-lengths, in millionths of a millimetre. 
wilh special reference to Coronce and Iridescent Clouds. 283

At the central point of the diffraction-pattern $(a \xi / f=0)$ the brightness is a maximum (instead of being zero as in the case of a thin plate, when $V=0$ ), and the colour-factor is $\lambda^{-2}$. About 250 the curve passes very near white, on the side towards blue-green. The colour then becomes yellowish, gradually improving, till at 450 a very fine orange is attained. And so on through the well-known series. When the retardation is large the curve approaches nearer and nearer to white, and, in the case of diffraction, the brightness diminishes indefinitely.

Along the diagonals of the pattern, where the colours are the purest, the factor is

$$
\lambda^{2} \sin \frac{\pi a \xi}{f \lambda} \text {. }
$$

I have determined two points on the curve, $\mathrm{D}_{1}$ in the first red $(a \xi / f=500)$, and $\mathrm{D}_{2}$ in the second green $(a \xi / f=810)$. The curve starts from the point $\lambda^{-2}$, and afterwards for several sweeps keeps outside of the thin-plate curve. The first orangeyellow and the second blue of the latter admit of but little improvement. But in the first red, which borders the central spot, the diagonals are far superior to the principal directions.

We now come to the main object of the present paper, the colours of iridescent clouds, formed by needles of ice. The colour-factor is

$$
\lambda \sin \frac{2 \pi a \xi}{f \lambda} \text {. }
$$

Comparing this with the factor for thin plates we see that the greater wave-lengths have an advantage. So the curve is, on the whole, displaced from the violet and towards the red. This curve is laid down on the diagram with a continuous line. Points actually determined are marked with small crosses, with the values of $a \xi / f$ annexed in bold figures*. The rest of the curve has been drawn by comparison with the thin-plate curve. The curve starts at the point $\lambda^{-1}$, so the central blue though bright is very impure. On the whole the colours are superior to those of thin plates. The reds are distinctly better, especially the third red. The second blue is nearly as good, though the third is decidedly inferior. The third and fourth greens are about on a par for purity, but more inclined to yellow, while the first and second yellows

- The calculations were inadvertently made for $\lambda^{2} \sin ^{2}(\pi a \xi / f \lambda)$, and the points given are put halfway between those thus found and the corresponding points on Lord Rayleigh's curve. All the calculations were made with a slide rule reading to $\frac{3}{3} \sigma$. 
are somewhat purer and more inclined to orange. The curve ultimately circles round closer and closer to the point $\lambda$, a very pale orange-yellow.

The custom of speaking of the successive diffraction spectra is apt to lead to the impression that each spectrum is purest in the middle when it does not overlap its neighbours. In the colours of the first two orders the exact contrary is the fact. $A$ better idea of the phenomenon is arrived at by considering the wave-lengths that are absent ; in other words, by considering the dark bands in the spectrum into which each colour could be drawn out. The fine yellows of the first two orders are due to the upper part of the spectrum being nearly quenched by broad dark bands, which as they proceed down the spectrum give the blues of the second and third orders. Before we can obtain a good green we must have two bands to blot out both ends of the spectrum. This occurs at 1330 and 1830 .

Fraunhofer (Verdet \& 70) using white light measured the deviations of the red bands in the diffraction-image of a slit, and, finding they were in the ratio $1: 2: 3 \ldots$, thought he had discovered the law for the successive maxima of homogeneous light. The complete explanation of this may be seen in the diagram; for the points 500,1000 , and 1500, corresponding to the absence of wave-lengths in the neighbourhood of 500, lie almost on the line from W to the red corner. The fourth red was probably not measured by Fraunhofer. The real maxima for wave-length 631 are at the points 0,900 , $1550,2190$.

Maxwell's colour diagram gives us complete information as to the hue and depth of each tint, but is silent as to the brightness; and with cloud colours, which are necessarily more or less contaminated with white light, the brightness is of great importance. It is clear, too, that the power of withstanding contamination depends on the depth as much as on the brightness. It occurred to me, therefore, that it would be instructive to draw a curve with retardations as abscissa, in which the ordinates should depend on both these qualities, and should represent what I will call the brilliancy of the colour. I have used the following principles:-(1) the brilliancy of white light is zero; (2) the brilliancy of standard red light is reckoned equal to that of standard green or violet light, when they are in the proportion in which they occur in white light; (3) the brilliancy of any colour which is composed of two standard colours is equal to the brilliancy of the more brilliant component. The third principle ensures that the brilliancy of complementary colours should be equal. As an 
with special reference to Coronce and Iridescent Clouds. 285

example of the application, suppose that the red, green, and violet components of two colours are $\cdot 536, \cdot 338, \cdot 042 ; \cdot 265$, $\cdot 114,084$ respectively. The components of white are $2 \cdot 01$, $3 \cdot 27,3 \cdot 22$. Reducing the first two triplets in proportion to the components of white, we have $\cdot 267, \cdot 104, \cdot 013 ; \cdot 132, \cdot 035$, -026. These may be considered as mixtures of two standard colours with some white light. Subtracting the white light, and taking the greater of the remaining components of each, we find the brilliancies are in the ratio $\cdot 254: \cdot 106$. Treated in this manner the brilliancies of the ten points in the spectrum I have chosen come out proportional to $20,47,39,30,18,26$, $31,19,10,5$. The maxima fall in the orange and the blueviolet. The intermediate minimum is in the green-blue, a part of the spectrum where the colour is generally considered poor. It seems probable that, when nearly swamped with white light, the colours would assert themselves nearly in proportion to their brilliancy. At the worst the brilliancy curves will be useful for comparing colours of similar hues.

In Plate $\mathrm{X}$. is given a curve representing the brilliancy of iridescent ice-clouds in accordance with the expression (6). $O$ wing to the occurrence of $\xi$ in the denominator the brilliancy is infinite when $\xi$ is zero, and decreases rapidly as $\xi$ increases. The ordinates of the latter portion of the curve are drawn on a scale tive times as great as those of the former. The points where the nearest approach is made to the pure colours of the spectrum are marked by the letter $p$. Under the most favourable circumstances in the clouds, when all the filaments are of the same size, there are two important causes of blurring. The first is the finite diameter of the sun, which, of course, prevents the brilliancy from being infinite. When the first purple is at $5^{\circ}$ from the sun-about an average distance-the colours over a range of 40 of retardation will be all mixed together. This effect will be less marked when the particles are finer and the colours further out. Another cause, more serious than the other, especially for large retardations, is the effective diameter of the filaments varying from 1 to $1 \cdot 155$.

Let us now deduce the successive colours from a study of the two diagrams, assuming the particles are of such a size as to give 100 of retardation to a degree of arc. Up to $1^{\circ}$ or $1 \frac{1}{2}^{\circ}$ from the centre of the sun the light is very bright and of a perceptibly bluish hue. If the cloud be dense even the face of the sun will be tinted blue. From $1 \frac{1}{2}^{\circ}$ to $21^{\circ}$ the light is practically white. Then a yellowish tinge asserts itself, which attains its greatest brilliancy at $3 \frac{1}{2}^{\circ}$ and its greatest purity at $4 \frac{1}{2}^{\circ}$. Between this and $5 \frac{1}{4}^{\circ}$ intervenes a narrow ring of Phil. Mag. S. 5. Vol. 28. No. 173. Oct. 1889. Y 


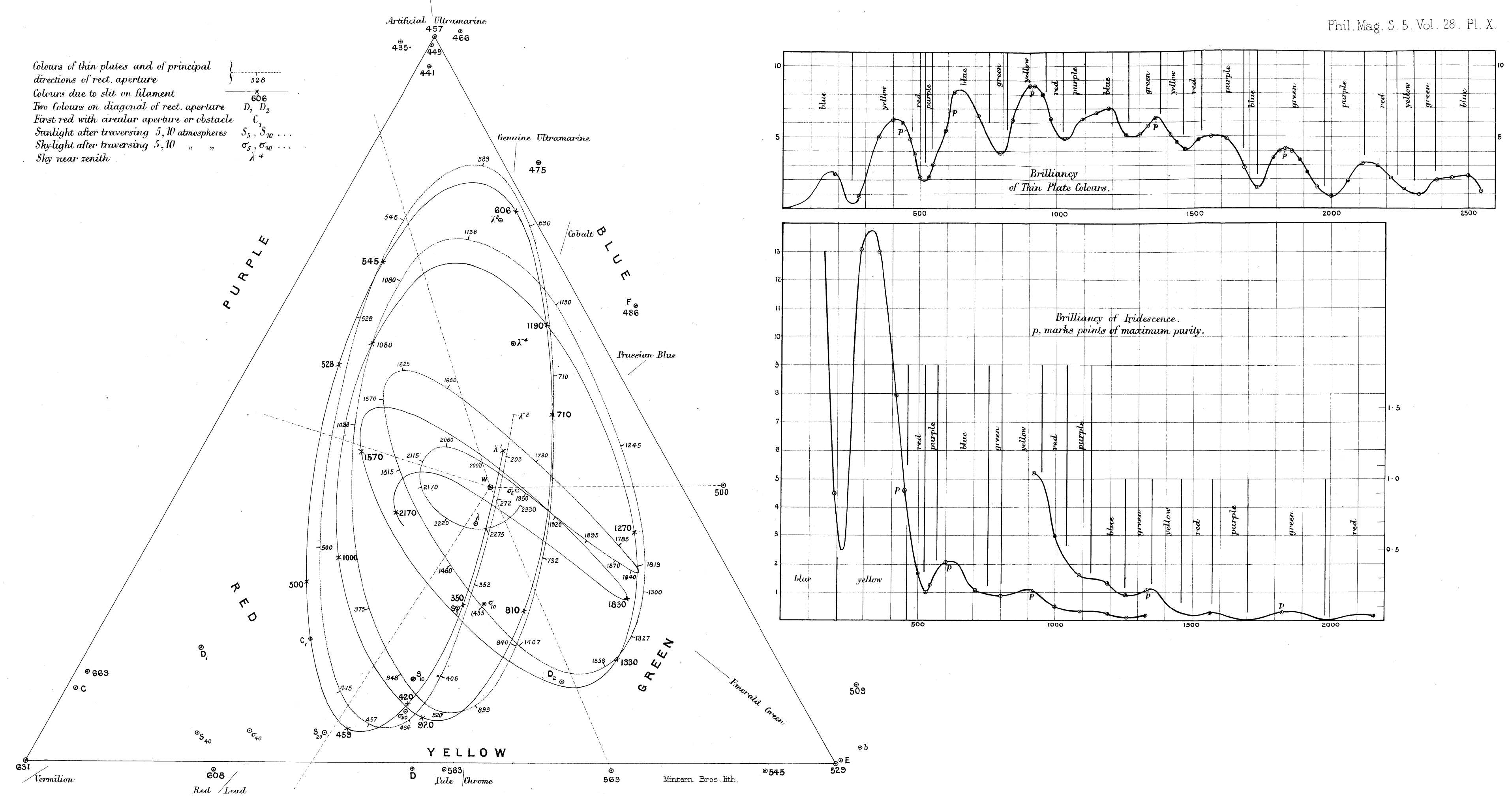


reddish orange. Then a bluish purple extends as far as $5 \frac{3}{4}^{\circ}$. A broad band of blue reaches to $7 \frac{1}{4}^{\circ}$, a poor green to $8 \frac{1}{4}^{\circ}$, a fine yellow to $9 \frac{1}{2}^{\circ}$, an orange-red to $10 \frac{1}{3}^{\circ}$, a reddish purple to $11 \frac{1}{4}^{\circ}$. The third blue (to $12 \frac{1}{2}^{\circ}$ ) is greener and decidedly poorer than the second. The third green (to $13 \frac{1}{2}^{\circ}$ ) is much inclined to yellow. The next noticeable colour is in the pink at $15 \frac{1}{2}^{\circ}$. There is a faint green at $18 \frac{1}{3}^{\circ}$, and a faint pink at $21 \frac{1}{2}^{\circ}$.

On Feb. 25th last I noted down some rather fine colours in ice-clouds, in which the tint seemed to depend mainly on the distance from the sun ; in order outwards, yellow, bright red, purple, green, greenish yellow, faint pink. A few minutes later the purple had altered to faint purple, bright blue, and the outer pink was succeeded by purple and green. This is a good illustration of the extent to which the theoretical colours are realized in observation.

Partly for the sake of comparison and partly on account of its intrinsic interest, I give the curve of brilliancy of thinplate colours, deduced from Lord Rayleigh's figures, with the addition of an ordinate I have calculated in the first blue. The light is supposed to fall at a uniform angle on a film of varying thickness.

When the diffracting particles are spherical the colourfactor is, as we have seen, $J_{1}^{2}(z)$. When $z$ is indefinitely small $\mathrm{J}_{1}(z)=z / 2$; so the curve starts from the point $\lambda^{-8}$. When $z$ is great

$$
\mathrm{J}_{1}^{2}(z)=2 \pi^{-1} z^{-1} \sin ^{2}\left(z-\frac{\pi}{4}\right)
$$

so the curve starts somewhat outside the filament curve and after a time comes near coincidence with it, finally oscillating about the same point $\lambda$. I have calculated the colour* for $2 \mathrm{R} r / f=600$. This is the point marked $\mathrm{C}_{1}$ on the diagram, which happens to fall exactly on the filament curve. I think we may conclude that from the first red upwards the colours produced by filaments and by drops will be practically identical.

In the previous section I have shown that for a not too small distance $\theta$ from the sun and for corresponding colours, i. e. when

$$
a \theta=2 \mathrm{R} \theta-\lambda / 4,
$$

the brightness of the water-cloud is about twice that of the ice-cloud. Thus we may make a fair approximation to the brilliancy curve of the former beyond the first purple by

* Using the table for $\phi(n)=2 n-1 \mathrm{~J}_{1}(n)$ given by Airy at the end of The Undulatory Theory of Optics.' 
with special reference to Corona and Iridescent Clouds. 287

letting the curve, hues included, stand as it is, and pushing the abscissæ to the left through a distance $\lambda / 4$. The quarter wave-length varies from 158 at the red corner to 114 at the violet corner; but it is sufficient to take the mean 136. Even in the first red we find $2 \mathrm{R} \theta=600$ corresponding to $a \theta=485$.

An easy way of seeing these colours to advantage is to lightly sprinkle the object-glasses of a pair of tield-glasses with lycopodium seed and direct them to the neighbourhood of the sun. 'The poorness of the green of the second order compared with that of the third order is well brought out, also the blueness of the first purple compared with the second. The green of the fourth order is quite distinct, and the corresponding red just visible.

The most notable difference between the colours of icefilaments and those of water-drops is the superiority of the first blue of the latter both in purity and extent. On the whole this agrees with observation, for the best inner blues that I have seen in water-clouds were superior to the best inner blues in ice-clouds.

\section{Colours of the Sky and Sun.}

To lend additional interest to the diagram I have calculated a few points representing these colours. It is now certain that the blue of the sky and the reddish tinge of the setting sun are mainly due to the scattering of light by particles small compared with a wave-length. The theory of this action is due to Lord Rayleigh *. All that we require for our present purpose is the law that the scattered light varies inversely as the fourth power of the wave-length. When the various parts of the spectrum are componnded in this proportion, we obtain the point marked $\lambda^{-4}$ on the diagram. This is a fair approximation to the blue of the sky near the zenith. Lord Rayleigh's preliminary measures gave the sky a somewhat richer hue.

Since the scattered light varies as $\lambda^{-4}$ it may be shown that the transmitted light must vary as $e^{-k x \lambda}-4$, where $x$ is the length of path and $k$ is a constant, depending on the size and material of the particles and on their number in a given space. The particles will, on the whole, be more numerous where the air is denser, and it is reasonable to take $x$ proportional to the mass of air traversed. Capt. Abney has found that if $x$ be expressed in atmospheres and $\lambda$ in thousandths of a millimetre $\left(\lambda_{D}=0.589\right), k$ has the value

* Phil. Mag. Feb., April, June, 1871, Aug. 1881. 
$0.013^{*}$. An atmosphere is defined to be the mass of air traversed by a line drawn vertically upwards from the level of the sea. The value depends on two series of observations on particularly fine days at South Kensington, when the airthicknesses were about 1.3 and 3.3 atmospheres. Taking the colour of the sun outside the atmosphere as the point $W$, the points $S_{5}, S_{10}, S_{20}, S_{40}$ on the diagram give the colour of sunlight which has traversed 5, 10, 20, and 40 atmospheres respectively. The first two are yellow inclining to orange, the third a fine orange, and the fourth redder than red lead. To the colour of the fourth, wave-lengths less than 529 contribute nothing appreciable; and even in the third the violet sensation is mainly due to wave-length 663 . For an observer at sea-level the first three thicknesses occur when the apparent zenith distance of the sun is $78 \frac{1}{2}^{\circ}, 85^{\circ}$, and $87 \frac{3}{4}^{\circ}$. For apparent Z.D. $90^{\circ}$ the thickness is 35.5 atmospheres $\dagger$. The additional 4.5 atmospheres can be secured by ascending a height of 330 feet, while from a height of 3000 feet the coloration due to 50 atmospheres can be studied. The same action is exhibited to some extent by clouds near the horizon and by distant snow mountains. For example, the Alps seen from Berne, forty miles away, look yellowish. But here the colour is interfered with by the intervening "blue sky." In other words, the particles, which sift the blue waves out of the light from the snow, send to the observer a by no means negligible quantity of scattered sunlight.

It is only when the colour of the sun is white that the sky is represented by $\lambda^{-4}$. If sunlight be represented by $S_{5}$, then skylight will be represented by $\sigma_{v}$, slightly on the green side of white. The paleness of the sky, when the sun is low, is a familiar phenomenon. Similarly $\sigma_{10}, \sigma_{20}$, correspond to $\mathrm{S}_{10}$, $\mathrm{S}_{20}$. But it is clearly of no consequence whether the shorter wave-lengths are filtered out before or after scattering. So if we could look at the ordinary blue sky through a tube, filled with air, 25 miles long it would appear pale greenish white. In the same way the blue of the sky near the horizon is of poorer quality than near the zenith. When the scattered light either before or after scattering has had to traverse 40 atmospheres, its colour reaches the point $\sigma_{40}$ on the diagram, i.e. it is really red. This is the red of a sunset sky. It is to be noticed that the form of the curves $W, S_{5}, S_{10}, S_{20}, S_{40}$, and

* Phil. Trans. 1887. His statements left me in some doubt as to the position of the decimal points, but the evidence of his diagram was decisive.

+ From Forbes's values, quoted by Abney, which allow for refraction and the curvature of the earth. 
$\lambda^{-4}, \sigma_{5}, \sigma_{10}, \sigma_{20}, \sigma_{40}$ is given by the theory, but to find the position of these points on the curves we require to know the value of $k$.

Since the triangle in my diagram is equilateral, the colour represented by any point $P$ within the triangle can be experimentally obtained in the following way:-Let a prismatic spectrum fall on a diaphragm with three adjustable slits, whose centres are at wave-lengths $63 \ddot{1}, 529$, and 457 . Make the breadths of the slits proportional to the perpendiculars drawn from $\mathrm{P}$ to the sides of the triangle. Then the three spectral rays, when compounded by a lens, will produce the colour P. The dispersion of Maxwell's spectrum is defined by the wave-lengths I have given of the sixteen equidistant points in his spectrum. In consequence, however, of individual variations in the colour sensations great accuracy would be thrown away.

Hotel Buol, Davos, July 10th.

XXXVII. On the Molecular Constitution of Isomeric Solutions \&.c. By Dr. G. Gore, F.R.S.*

TN the present research, the "voltaic balance" has been 1 applied to the detection of differences of chemical constitution of a pair of isomeric solutions; and to detect molecular and chemical changes in them, caused by heat, light, lapse of time, order of mixture, degree of dilution of ingredients, \&c.

According to the results of J. Thomsen's thermochemical investigations, as described by $\mathrm{P}$. Muir ('Principles of Chemistry,' 1884, pp. 434,437), "when nitric acid and sodium sulphate react in equivalent quantities in a dilute aqueous solution, heat is absorbed; but when sulphuric acid and sodium nitrate react under similar conditions, heat is evolved. But the final distribution of the base between the two acids will be the same in both cases, and, moreover, this distribution will be the same when equivalent quantities of the two acids (sulphuric and nitric) and the base (soda) mutually react." "When soda, nitric acid, and sulphuric acid mutually react in equivalent quantities in a dilute aqueous solution, two thirds of the soda combines with the nitric acid, and one third with the sulphuric acid." "The final division of the base between the two acids is the same whether the soda were originally present as sulphate or nitrate." (See also "Theories

* Communicated by the Author. 